\title{
Decreased calpain 6 expression is associated with tumorigenesis and poor prognosis in HNSCC
}

\author{
YINZHOU XIANG $^{1 *}$, FEN LI $^{1,2^{*}}$, LEI WANG $^{1}$, ANYUAN ZHENG $^{1}$, JINGJING ZUO $^{1}$, MAN LI $^{1}$, \\ YONGPING WANG ${ }^{1}$, YONG XU ${ }^{1}$, CHEN CHEN $^{1,2},{\text { SHIMING } \text { CHEN }^{1,2}, \text { BOKUI XIAO }^{1,2} \text { and ZEZHANG TAO }}^{1,2}$ \\ ${ }^{1}$ Department of Otolaryngology-Head and Neck Surgery; ${ }^{2}$ Research Institute of Otolaryngology-Head and Neck Surgery, \\ Renmin Hospital of Wuhan University, Wuhan, Hubei 430060, P.R. China
}

Received January 27, 2016; Accepted November 25, 2016

DOI: $10.3892 / \mathrm{ol} .2017 .5687$

\begin{abstract}
Calpains are a family of intracellular cysteine proteases involved in various biological processes. Previously, the family was identified to have abnormal expression in several types of malignant tumor. Calpain 6 was less well known; however, it was recently identified to be involved in the carcinogenesis of certain types of malignant tumor. However, the expression of calpain 6 in head and neck squamous cell carcinoma (HNSCC) remains unclear. A total of six datasets from the Gene Expression Omnibus (GEO) was analyzed and an association between calpain 6 expression levels and HNSCC was identified, with the expression of calpain 6 observed to be significantly decreased in HNSCC $(\mathrm{P}<0.01)$. However, the expression of calpain 6 may vary between distinct tumor stages of HNSCC. Furthermore, calpain 6 expression was positively associated with the survival rate in patients with HNSCC $(\mathrm{P}<0.05)$, with increased expression of calpain 6 associated with an improved survival outcome. Calpain 6 expression was analyzed using an HNSCC tissue microarray and these results were consistent with the statistical analysis of the bioinformatics data from the GEO, indicating that calpain 6 may be a tumor suppressor protein in HNSCC.
\end{abstract}

\section{Introduction}

Head and neck squamous cell carcinoma (HNSCC) is a type of malignant epithelial tumor that occurs as a result of the

Correspondence to: Professor Zezhang Tao, Department of Otolaryngology-Head and Neck Surgery, Renmin Hospital of Wuhan University, 238 Jie-Fang Road, Wuhan, Hubei 430060, P.R. China E-mail: taozezhang@hotmail.com

Dr Chen Chen, Research Institute of Otolaryngology-Head and Neck Surgery, Renmin Hospital of Wuhan University, 238 Jie-Fang Road, Wuhan, Hubei 430060, P.R. China

E-mail: chenchen_md@whu.edu.cn

*Contributed equally

Key words: head and neck squamous cell carcinoma, calpain 6, Gene Expression Omnibus, tissue microarray, tumor stage dysregulation of multiple genes $(1,2)$. The underlying pathological mechanisms involved are complex and remain unclear. Recently, the incidence of HNSCC has increased; furthermore, the survival rate and prognosis of patients is poor, particularly when local lymph node metastasis has occurred (3). The identification of the underlying molecular mechanisms and development of more effective adjuvant therapies is warranted.

Calpains were identified as a family of intracellular cysteine proteases, whose functions are dependent on the concentration of $\mathrm{Ca}^{2+}(4,5)$. Calpains were identified to be involved in various biological processes, including cytoskeleton remodeling, signal transduction and cell apoptosis (6-8). Currently, 16 family members have been identified (9), which are classified into two types: The classical calpains and the non-classical calpains $(4,5,10-15)$. Due to slight variations in their structure, the various calpains were identified to be associated with various physiological activities.

Calpain 6 was identified to be a non-classical calpain, coded for by a gene located on the $\mathrm{X}$ chromosome (16). Active calpains are heterodimers comprising a catalytic subunit and a regulatory subunit (9). The catalytic subunit is the larger subunit $(80 \mathrm{kDa})$, which contains four domains: The $\mathrm{N}$-terminal anchor helix region; the conserved CysPc catalytic domain, composed of two protease core (PC) domains (PC1 and $\mathrm{PC} 2$ ); the calpain type $\beta$-sandwich (CBSW) domain, which undergoes a conformational change when bound to calcium; and the penta-EF-hand (PEF) domain (9). The regulatory subunit is the smaller subunit $(30 \mathrm{kDa})$, coded for by the calpain small subunit 1 gene (also known as calpain 4), which contains two domains (9). Although calpain 6 is a type of non-classical calpain $(7,10)$, its catalytic subunit contains only the CBSW domain and not the classical PEF domain $(7,10)$. Calpain 6 also lacks an active catalytic cysteine residue in the $\mathrm{PC} 1$ domain, which is replaced by lysine, resulting in it being devoid of proteolytic activity $(7,10)$. Furthermore, calpastatin, an inhibitor of the classical calpains, has no effect on calpain $6(7,10)$.

Calpains were identified to exhibit abnormal expression in several types of malignant tumor, including schwannoma, breast cancer, gastric cancer, colorectal cancer, melanoma, pancreatic cancer, laryngeal carcinoma and endometrial carcinoma $(9,11,12,14,17-20)$. Compared with other calpain family members, calpain 6 is less well known; however, it was 
revealed to be associated with the carcinogenesis of certain types of malignant tumor. Lee et al (21) reported calpain 6 overexpression in uterine sarcoma, carcinoma and uterine fibroids. The expression characteristic of calpain 6 in HNSCC remains to be reported. In the present study, data from the Gene Expression Omnibus (GEO) were analyzed, and it was identified that the expression of calpain 6 was associated with the occurrence of HNSCC. HNSCC tissues were also collected for further verification.

\section{Materials and methods}

Datasets. The GEO repository of the National Center for Biotechnology Information (Bethesda, MD, USA; www.ncbi. nlm.nih.gov/geo) is a public functional genomics data repository. In order to investigate the potential association between the expression of calpain 6 and the occurrence of HNSCC, six datasets were retrieved from the GEO and were subjected to data analysis. The gene expression data series (GSE) of human HNSCC tissues, benign lesions of the head and neck, and wild-type tissues, included the GSE33205, GSE55550, GSE59102, GSE39366, GSE65858 and GSE27020 datasets. The early stage and advanced stage mentioned in dataset GSE59102 refer to tumor stage (T)1/T2 and T3/T4, respectively. The analysis of the potential association between the expression of calpain 6 and the occurrence of HNSCC was conducted on each of the six datasets independently.

Tissue microarray (TMA). The HN803b TMA was purchased from US Biomax, Inc. (Derwood, MD, USA). The TMA consisted of three types of tumor, including tongue carcinoma, larynx carcinoma and nose carcinoma, and wild-type tissues. A total of 62 males and 18 females formed the group. The mean age was 53.4 years (range, 18-90 years). The number of cases of grades 1, 2 and 3 were 10, 34 and 21 respectively; the number of cases of $\mathrm{T}$ stages 1,2,3 and 4 were 5,33,15 and 9, respectively; and the number of cases of node $(\mathrm{N})$ stages 0,1 and 2 were 38, 16 and 3, respectively.

Immunohistochemistry $(\mathrm{IHC})$. The expression of calpain 6 in the TMA was detected using IHC. The IHC was performed using a two-step method with an IHC kit (Guge Biotechnology Co., Ltd., Wuhan, China), according to the manufacturer's protocol. The TMA was deparaffinized and rehydrated at room temperature with the following treatments: Xylene I, $5 \mathrm{~min}$; xylene II, $5 \mathrm{~min}$; $100 \%$ ethanol, $5 \mathrm{~min}$; $95 \%$ ethanol, $5 \mathrm{~min}$; 85\% ethanol, $5 \mathrm{~min}$; $70 \%$ ethanol, $5 \mathrm{~min}$; distilled water, 5 min. Citric acid buffer solution ( $\mathrm{pH} 6.0$ ) was used for antigen retrieval in a microwave. To block endogenous peroxidase activity, the TMA was immersed in $3 \% \mathrm{H}_{2} \mathrm{O}_{2}$ for $10 \mathrm{~min}$, and incubated with anti-calpain 6 primary antibody (1:100 dilution; cat. no. HPA005992; Sigma-Aldrich; Merck Millipore, Darmstadt, Germany) overnight at $4^{\circ} \mathrm{C}$. The TMA was incubated with poly-horseradish peroxidase-conjugated goat anti-rabbit secondary antibody (cat. no. BA1039; Boster Biological Technology, Wuhan, China) for $30 \mathrm{~min}$ at $37^{\circ} \mathrm{C}$ (following each of the steps, the TMA was washed three times with PBS for $5 \mathrm{~min}$ ), stained with diaminobenzidine at room temperature for $5 \mathrm{~min}$ and then stained with hematoxylin at room temperature for $2 \mathrm{~min}$. Finally, the TMA was dehydrated and mounted with neutral balata, then observed and imaged using a BX40 microscope (Olympus Corporation, Tokyo, Japan) and the CC-12 Soft-Imaging System (Olympus Soft Imaging Solutions GmbH, Münster, Germany).

Evaluation of IHC staining. The evaluation of calpain 6 expression was quantified and scored for intensity (range, 0-3) and frequency (range, $0-4$ ). The intensity was referred to as follows: Grade (0), negative staining; grade (1), weak staining intensity; grade (2), moderate staining intensity; or grade (3), strong staining intensity. The frequency scores were divided into four grades (0-25, 26-50, 51-75 and 76-100\%) according to the number of positively stained tumor cells. The composite expression score (CES) was calculated as follows: Intensity $x$ frequency. The CES was scored (range, $0-12$ ) as follows: Negative (0); weak positive (1-4); positive (5-8); or strong positive (9-12).

Statistical analysis. Gene expression profiling data from the GEO were imported into Microsoft Excel 2007 (Microsoft Corporation, Redmond, WA, USA). Results are presented as the mean \pm standard deviation. All statistical analyses were performed using SPSS software (version 16.0; IBM SPSS, Armonk, NY, USA). Calpain 6 expression in primary tumors was analyzed using a $\chi^{2}$ test, Fisher's exact test and independent samples t-test. The survival time of patients with calpain 6 positive and negative expression status was compared with the survival analysis. Calpain 6 expression in the TMA was analyzed using one-way analysis of variance and Bonferroni's multiple comparison tests among groups. $\mathrm{P}<0.05$ was considered to indicate a statistically significant difference.

\section{Results}

Calpain 6 expression is significantly decreased in HNSCC. The GSE33205 dataset originated from 44 HNSCC tumor and 25 wild-type mucosa tissue samples, and the wild-type samples derived from a uvulopalatopharyngoplasty. The expression of calpain 6 was significantly decreased in HNSCC tissue compared with wild-type tissue ( $\mathrm{P}=0.0001$; Fig. 1A).

The GSE59102 dataset originated from 29 laryngeal squamous cell carcinoma (LSCC) cancer and 13 marginal tissue samples, which were collected from patients undergoing surgical ablation of LSCC. The expression of calpain 6 was significantly decreased in LSCC tissue (early-stage and advanced stage) compared with marginal tissue (early stage, $\mathrm{P}=0.0193$; advanced stage, $\mathrm{P}=0.0372$, Fig. 1B).

The GSE55550 dataset originated from 56 tumor and 9 benign tissue samples. The tumor tissue samples consisted of 38 oropharyngeal cancer samples, 16 oral cancer samples, 1 hypopharyngeal carcinoma sample and 1 maxillary carcinoma sample. The nine benign samples were wild-type benign uvula tissue samples collected from patients with sleep apnea. The expression of calpain 6 was significantly decreased in tumor tissue compared with benign tissue ( $\mathrm{P}=0.0008$; Fig. 1C). These data suggest that the expression of calpain 6 was significantly different between HNSCC tissue and wild-type tissue.

Calpain 6 expression is not significantly associated with HNSCC tumor grade. The GSE39366 dataset originated from 

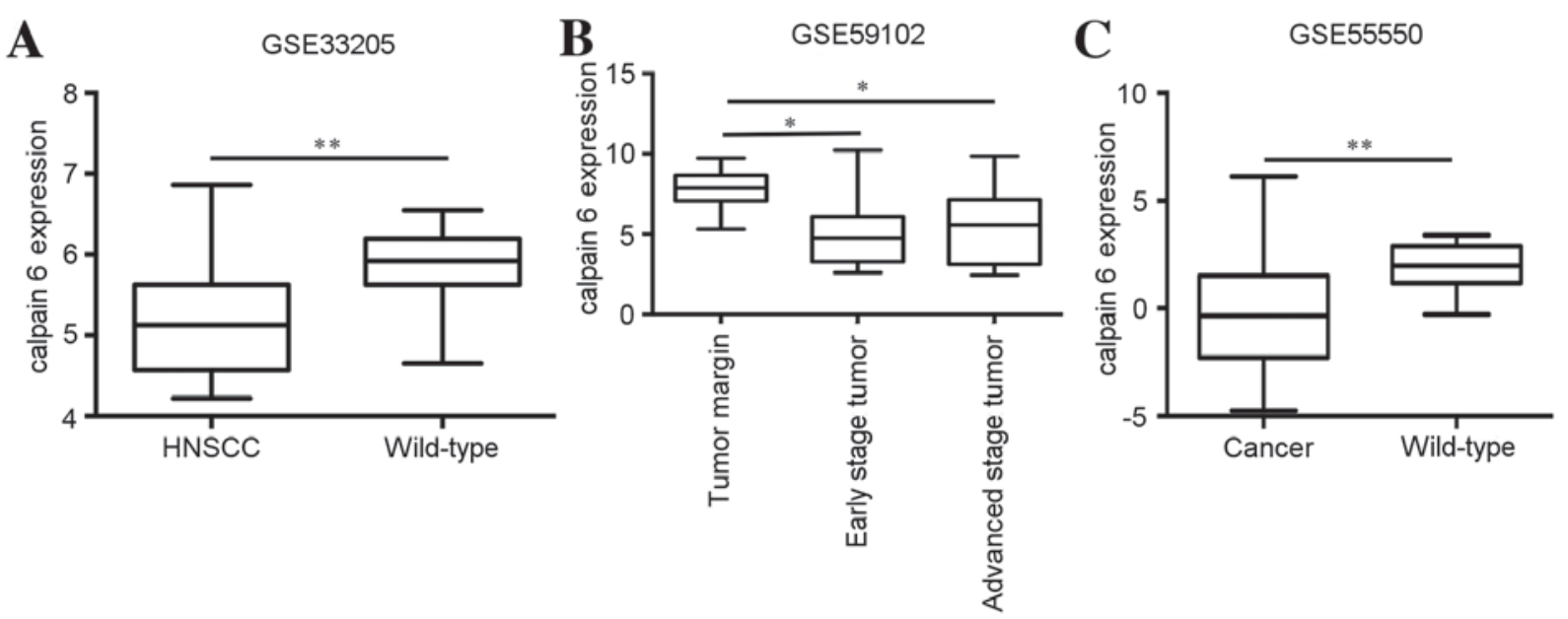

Figure 1. Expression of calpain 6 is decreased in HNSCC. A total of three datasets were obtained from the Gene Expression Omnibus for analysis of calpain 6 expression: (A) GSE33205, (B) GSE59102 and (C) GSE55550. * $\mathrm{P}<0.05$; ${ }^{* *} \mathrm{P}<0.01$. HNSCC, head and neck squamous cell carcinoma; GSE, gene expression data series.

a total of 138 HNSCC samples. No significant difference in the expression of calpain 6 among the distinct tumor grades was identified ( $\mathrm{P}=0.0644$; Fig. 2A).

The GSE27020 dataset originated from 59 fresh-frozen laryngeal cancer tissues. No significant difference in the expression of calpain 6 among the distinct tumor grades was identified ( $\mathrm{P}=0.0703$; Fig. 2B).

These data suggest that the expression of calpain 6 was not significantly different among distinct HNSCC tumor grades.

Calpain 6 expression is not significantly associated with HNSCC N stage. For the GSE65858 dataset, no significant difference in the expression of calpain 6 among distinct $\mathrm{N}$ stages in HNSCC was identified ( $\mathrm{P}=0.0813$; Fig. 2C). For the GSE39366 dataset, no significant difference in the expression of calpain 6 among the distinct $\mathrm{N}$ stages in HNSCC was identified ( $\mathrm{P}=0.1014$; Fig. 2D). These data suggest that the expression of calpain 6 was not significantly different among distinct $\mathrm{N}$ stages of HNSCC.

Calpain 6 expression may be significantly associated with HNSCC T stage. The GSE65858 dataset originated from $270 \mathrm{HNSCC}$ patient samples. A significant difference in the expression of calpain 6 among distinct T stages in HNSCC was identified ( $\mathrm{P}=0.0152$; Fig. $3 \mathrm{~A})$.

For the GSE39366 dataset, no significant difference in the expression of calpain 6 among distinct T stages in HNSCC was identified ( $\mathrm{P}=0.0561$; Fig. 3B).

These data suggest that the expression of calpain 6 may be significantly different among distinct $\mathrm{T}$ stages of HNSCC; however, this remains unclear.

Calpain 6 expression is significantly associated with the survival rate of patients with HNSCC. For the GSE27020 dataset, a significant association between the high (positive expression status) and low (negative expression stats) expression of calpain 6 and disease-free survival rate (DFS) in HNSCC was identified ( $\mathrm{P}=0.0295$; Fig. 3C). These data suggest that the HNSCC patients with increased expression of calpain 6 exhibited improved survival rates.
Calpain 6 expression is not significantly associated with the degree of tumor differentiation in HNSCC tissue. To further study the effect of calpain 6 expression in HNSCC, an HNSCC TMA was used. IHC staining was used for the detection of calpain 6 (Fig. 4A). The expression of calpain 6, measured as the CES, was significantly decreased in tumor tissues of each grade compared with wild-type tissue ( $\mathrm{P}=0.0374$; Fig. $4 \mathrm{~B})$. No significant difference in the expression of calpain 6 among distinct grades of tumor differentiation was identified in the TMA ( $\mathrm{P}=0.1429$, Fig. 4B).

Calpain 6 expression is significantly associated with $T$ stage in HNSCC tissue. To investigate the association between the expression of calpain 6 and the T/N stage of HNSCC, an HNSCC TMA was analyzed. IHC staining was used for the detection of calpain 6 (Fig. 4C). The expression of calpain 6, determined as the CES, was significantly decreased in tumor tissues at each $\mathrm{T}$ stage compared with wild-type tissue ( $\mathrm{P}=0.0353$; Fig. 4D), and the expression of calpain 6 was significantly different between stages T1/T2 and T3/T4 ( $\mathrm{P}=0.0266$; Fig. 4D); however, no significant difference between stages $\mathrm{T} 1$ and $\mathrm{T} 2(\mathrm{P}=0.0737)$, or between stages T3 and T4 (P=0.0619) was identified (Fig. 4D).

Calpain 6 expression is not significantly associated with $N$ stage in HNSCC tissue. The HNSCC TMA was used to investigate the potential association between the expression of calpain 6 and the $\mathrm{N}$ stage of HNSCC. IHC staining was used for the detection of calpain 6 (Fig. 4E). The expression of calpain 6, determined as the CES, was significantly decreased in tumor tissues at each $\mathrm{N}$ stage compared with wild-type tissue $(\mathrm{P}=0.0437$; Fig. $4 \mathrm{~F})$, whereas the difference in calpain 6 expression between stages N0, N1 and N2 was not statistically significant ( $\mathrm{P}=0.0866$; Fig. 4F).

\section{Discussion}

The calpain family was initially identified in rat brain tissue in 1964 (16,22). Subsequently, calpains have been extensively studied, and family members were reported to serve important roles in multiple processes and diseases, including cytoskeleton 

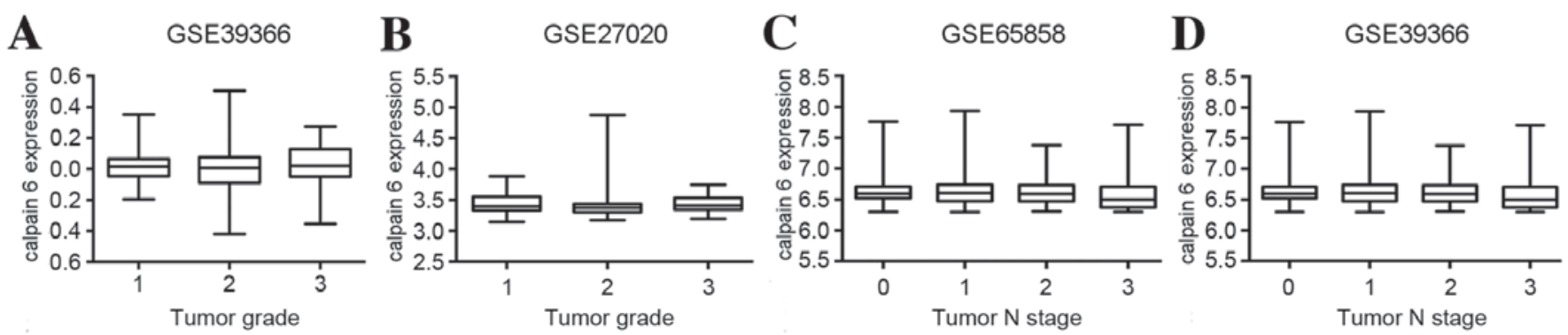

Figure 2. Expression of calpain 6 is not significantly associated with tumor grade or N stage in head and neck squamous cell carcinoma. A total of two datasets were obtained from the Gene Expression Omnibus for analysis of calpain 6 expression in association with tumor grade: (A) GSE39366 and (B) GSE27020. A total of two datasets were obtained from the Gene Expression Omnibus for analysis of calpain 6 expression in association with N stage: (C) GSE65858 and (D) GSE39366. GSE, gene expression data series; N, node.
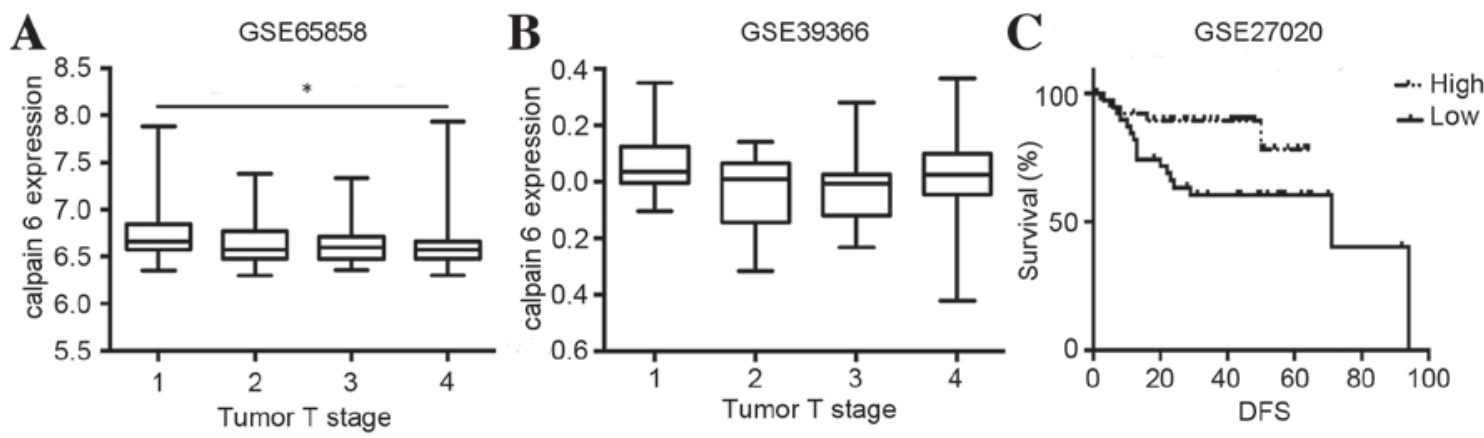

Figure 3. Expression of calpain 6 may be associated with the T stage of HNSCC and patient survival. Datasets were obtained from the Gene Expression Omnibus and differences in the expression of calpain 6 in different T stages of HNSCC were analyzed. (A) GSE65858. (B) GSE39366. (C) A Kaplan-Meier survival curve was produced from GSE27020. ${ }^{*} \mathrm{P}<0.05$ between stages 1, 2, 3 and 4. HNSCC, head and neck squamous cell carcinoma; GSE, gene expression data series; T, tumor; DFS, disease-free survival rate.

remodeling, signal transduction, cell apoptosis, the generation and development of neurodegenerative diseases, diabetic polyneuropathy and myodystrophy $(5,6,8,11,23,24)$. Consistent with the increasing number of studies on calpains associated with malignant tumors, the involvement of calpains in the carcinogenesis of several types of cancer has been identified (14,17,19-21,25-29).

Calpain 6 was recently reported to be overexpressed in uterine stromal sarcoma, carcinosarcoma, cervical neoplasia and osteosarcoma $(25,26)$. Calpain 6 was predominantly expressed in embryonic striated muscle and in placenta, and in adult cells its expression was low (8). Furthermore, the expression of calpain 6 increased in association with the progression of cervical cancer (25). Lee et al (25) identified that calpain 6 expression was significantly increased during the progression from low-grade squamous intraepithelial lesions (cervical intraepithelial neoplasia) to invasive squamous cell carcinomas with lymph node metastases.

In the present study, the potential association of calpain 6 with tumorigenesis of HNSCC was investigated. Initially, three large independent HNSCC datasets were analyzed. GSE33205 (44 HNSCC tumor tissues and 25 wild-type mucosa samples), GSE55550 (56 tumor tissue samples and 9 benign samples) and GSE59102 (29 cancer samples and 13 marginal samples) were obtained from the GEO repository; three independent statistical analyses between the tumor tissues and the wild-type tissues were performed. It was concluded that the expression of calpain 6 was significantly decreased in HNSCC tissues compared with wild-type tissues. It was hypothesized that calpain 6 was a tumor suppressor protein in HNSCC, although the results of the present study were not consistent with the results from previous studies on cervical cancer $(25,26)$. Calpain 6 has been less frequently reported in tumor-associated studies, and the expression patterns of calpain 6 in tumors other than HNSCC remain unclear. The reason for this discrepancy is unknown; however, it was hypothesized that it may be due to the location of the tumor, the progenitor cells and the associated environmental factors $(30,31)$. Further research is required to elucidate the reasons for this difference.

In the present study, the potential correlation between the expression of calpain 6 and tumor grade and tumor stage was analyzed. No significant difference in calpain 6 expression among tumor grades 1, 2 and 3 was identified; however, a significant difference in calpain 6 expression between $T$ stages was identified for one dataset (GSE65858), but not another (GSE39366), suggesting that the expression of calpain 6 may be different between distinct $\mathrm{T}$ stages; however, this remains unclear.

An HNSCC TMA was analyzed and IHC staining was used for the detection of calpain 6 (Fig. 4). The expression of calpain 6 was significantly decreased in tumor tissues compared with wild-type tissue; furthermore, the expression of calpain 6 was significantly decreased in T3 and T4 compared with T1 and T2. The expression of calpain 6 was significantly decreased in tumor tissues at each $\mathrm{N}$ stage 

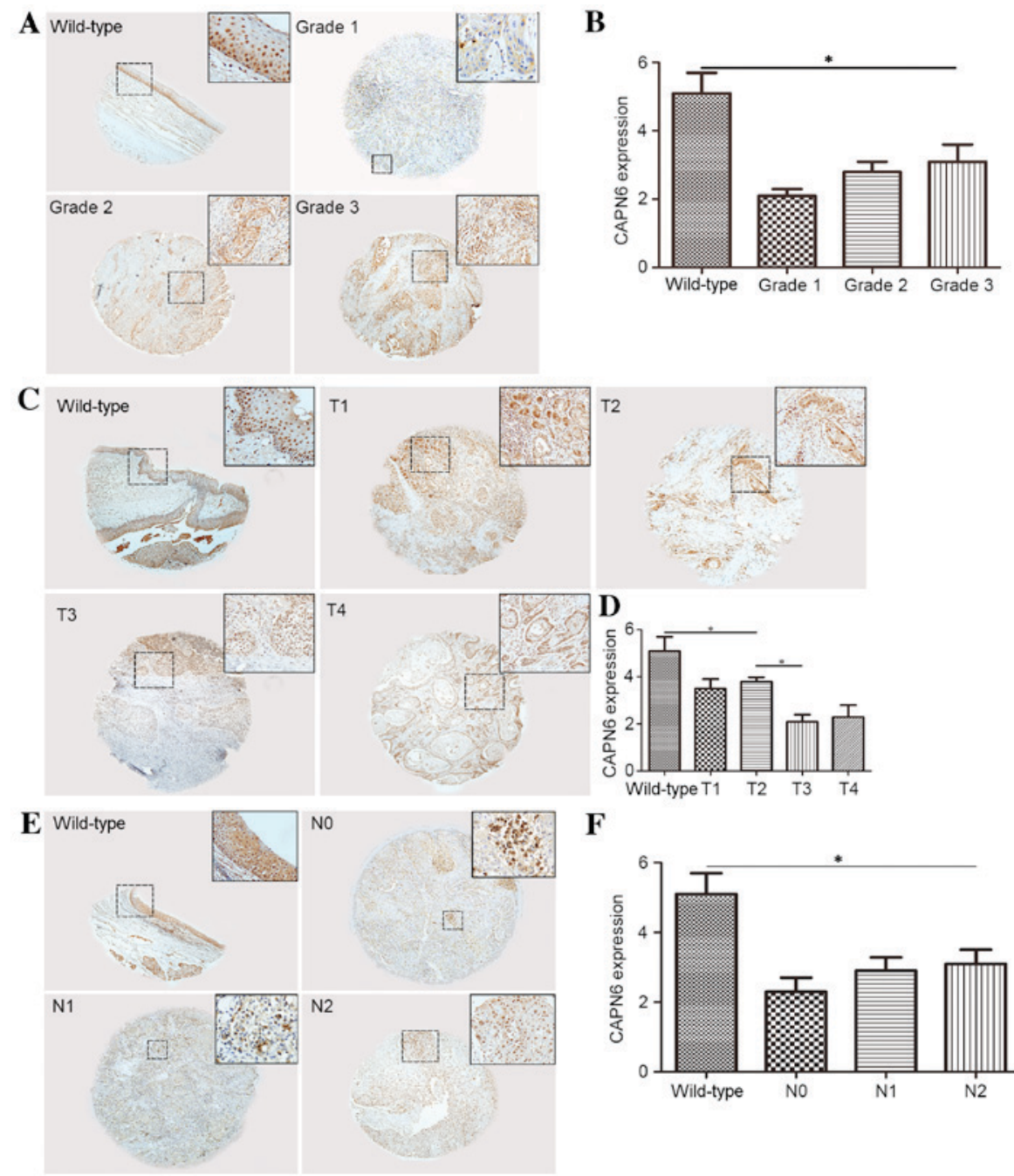

$\mathbf{F}$

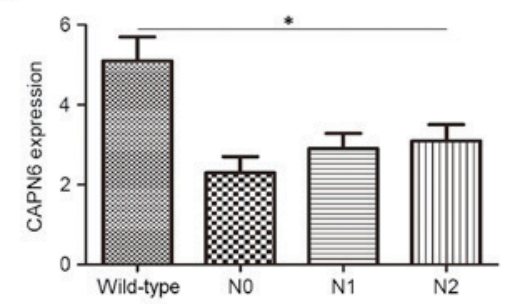

Figure 4. Expression of calpain 6 in HNSCC TMA. IHC staining was used for the detection of calpain 6. (A) No difference in IHC staining of calpain 6 was revealed among distinct tumor grades of HNSCC in the TMA. (B) Calpain 6 expression, determined as the CES, was decreased in tumor tissues. (C) IHC staining of calpain 6 appeared to vary among distinct T stages of HNSCC in the TMA. (D) Calpain 6 expression, determined as the CES, was decreased in tumor tissues. (E) No difference in the IHC staining of calpain 6 was revealed among distinct N stages of HNSCC in the TMA. (F) Calpain 6 expression, determined as the CES, was decreased in tumor tissues. "P<0.05. HNSCC, head and neck squamous cell carcinoma; TMA, tissue microarray; IHC, immunohistochemical; CES, composite expression score; N, node; T, tumor; CAPN6, calpain 6.

compared with wild-type tissue; however, the difference in calpain 6 expression among N0, N1 and N2 stages was not statistically significant. These results suggested that calpain 6 expression may be associated with tumorigenesis in HNSCC. Conversely, tumor growth or metastasis may be the reason for the downregulation of calpain 6 in HNSCC. According to this hypothesis, calpain 6 may serve a role in the prevention and management of HNSCC. Furthermore, calpain 6 may be considered to be a tumor biomarker in HNSCC. Further research is required to elucidate the role and underlying molecular mechanism of calpain 6 in cancer prevention, treatment and prognosis.

As calpain 6 was speculated to be a tumor suppressor protein, further statistical analysis of calpain 6 and survival estimators in HNSCC patients was carried out. The GSE27020 dataset (which originated from 59 fresh-frozen laryngeal cancer tissues) was used for the statistical analysis of the expression of calpain 6 and the DFS in HNSCC. The data suggested that the HNSCC patients with increased expression of calpain 6 had improved survival rates.
In previous studies, calpains have been identified to be associated with several types of malignant tumor $(6,12-14,17,18,24)$. Although the association between calpains and cancer has been investigated, the underlying molecular mechanisms remain unclear. In addition, several studies $(13,20,32,33)$ using calpain inhibitors were conducted. The calpain inhibitor PD150606 was used in pancreatic cancer cells, as soy isoflavones and calpain inhibition have been suggested to exert inhibitory effects on cancer development and progression (20). Rothe et al (20) tested and identified that the anticancer activity of the beverage Haelan 951 may be potentiated when administered with calpain inhibitors.

Several studies have focused on the association of calpain 6 with chemoresistance. Rho et al (26) overexpressed calpain 6 or inhibited calpain 6 in HeLa cells and human umbilical vein endothelial cells, evaluated the levels of cisplatin-mediated apoptosis and vascular endothelial growth factor-mediated angiogenesis, and concluded that calpain 6 induced tumorigenesis by inhibiting apoptosis and facilitating angiogenesis. Tonami et al (23) reported that, upon interaction 
with guanine-nucleotide-exchange factor $\mathrm{H} 1$, calpain 6 regulated Ras-related C3 botulinum toxin substrate 1 activity and cell motility.

The calpains were identified as a family of cysteine proteases that are expressed in various tissues $(9,11)$. Numerous proteins have been revealed to be hydrolyzed by calpains; however, calpain 6 was identified to be a non-proteolytic family member. To date, the development of drugs to promote the inhibition of calpains has focused on targeting the proteolytic function of calpains (34). The calpains may hydrolyze nuclear factor $x \mathrm{~B}$ inhibitor or Myc proto-oncogene protein to promote cell survival and decrease the response to apoptotic signaling; alternatively, several kinases may be hydrolyzed by calpains to promote cell migration, which may be associated with cancer metastasis (34). Calpain 6 has been less well studied owing to its non-classical structure. Liu et al (7) reported that microRNA 449a may downregulate calpain 6 and POU domain class 2 transcription factor 1 (POU2F1) to promote liver cancer cell apoptosis; Liu et al (7) explored the association between the expression of calpain 6 and protein kinase B in several cell lines, including HeLa, human embryonic kidney-293T, A549 and the liver cancer cell line 7404, and they identified that the phosphoinositide 3-kinase-protein kinase B signaling pathway may upregulate calpain 6 gene promoter activity. Furthermore, activator protein 1, forkhead box D3 and POU2F1 were also revealed to be able to regulate calpain 6 expression and its activity.

The results of the present study confirmed the important role of calpain 6 in HNSCC, as the expression of calpain 6 was significantly decreased in HNSCC tissues compared with the wild-type tissues; in addition, the survival proportions of HNSCC patients were statistically increased in the calpain 6 high expression group compared with the low expression group. Furthermore, the results from the TMA of HNSCC patients were similar. The exact role of calpain 6 association with HNSCC carcinogenesis remains unclear, and the net effects of calpain 6 in HNSCC also require further research.

\section{Acknowledgements}

The present study was supported by the National Natural Science Foundation of China (grant no. 81372880), the doctoral program of the Higher Education Research Fund (grant nos. 20130141120093 and 20110141110062) and the Natural Science Foundation of Hubei Province (grant no. 2012FFA045).

\section{References}

1. Sacco AG and Cohen EE: Current treatment options for recurrent or metastatic head and neck squamous cell carcinoma. J Clin Oncol 33: 3305-3313, 2015.

2. Marur S and Forastiere AA: Head and neck squamous cell carcinoma: Update on epidemiology, diagnosis, and treatment. Mayo Clin Proc 91: 386-396, 2016.

3. de Bree R, Takes RP, Castelijns JA, Medina JE, Stoeckli SJ, Mancuso AA, Hunt JL, Rodrigo JP, Triantafyllou A, Teymoortash A, et al: Advances in diagnostic modalities to detect occult lymph node metastases in head and neck squamous cell carcinoma. Head Neck 37: 1829-1839, 2015.

4. Croall DE and Ersfeld K: The calpains: Modular designs and functional diversity. Genome Biol 8: 218, 2007.
5. Hanna RA, Campbell RL and Davies PL: Calcium-bound structure of calpain and its mechanism of inhibition by calpastatin. Nature 456: 409-412, 2008

6. Hata S, Abe M, Suzuki H, Kitamura F, Toyama-Sorimachi N Abe K, Sakimura K and Sorimachi H: Calpain $8 / \mathrm{nCL}-2$ and calpain 9/nCL-4 constitute an active protease complex, G-calpain, involved in gastric mucosal defense. PLoS Genet 6: e1001040, 2010.

7. Liu Y, Mei C, Sun L, Li X, Liu M, Wang L, Li Z, Yin P, Zhao C, Shi Y, et al: The PI3K-Akt pathway regulates calpain 6 expression, proliferation, and apoptosis. Cell Signal 23: 827-836, 2011.

8. Tonami K, Hata S, Ojima K, Ono Y, Kurihara Y, Amano T, Sato T, Kawamura Y, Kurihara H and Sorimachi H: Calpain-6 deficiency promotes skeletal muscle development and regeneration. PLoS Genet 9: e1003668, 2013.

9. Moretti D, Del Bello B, Allavena G and Maellaro E: Calpains and cancer: Friends or enemies? Arch Biochem Biophys 564: 26-36, 2014.

10. Tonami K, Kurihara Y, Aburatani H, Uchijima Y, Asano T and Kurihara H: Calpain 6 is involved in microtubule stabilization and cytoskeletal organization. Mol Cell Biol 27: 2548-2561, 2007.

11. Storr SJ, Carragher NO, Frame MC, Parr T and Martin SG: The calpain system and cancer. Nat Rev Cancer 11: 364-374, 2011.

12. Storr SJ, Lee KW, Woolston CM, Safuan S, Green AR, Macmillan RD, Benhasouna A, Parr T, Ellis IO and Martin SG: Calpain system protein expression in basal-like and triple-negative invasive breast cancer. Ann Oncol 23: 2289-2296, 2012.

13. Fei B, Yu S and Geahlen RL: Modulation by Syk of Bcl-2, calcium and the calpain-calpastatin proteolytic system in human breast cancer cells. Biochim Biophys Acta 1833: 2153-2164, 2013.

14. Storr SJ, Pu X, Davis J, Lobo D, Reece-Smith AM, Parsons SL, Madhusudan S and Martin SG: Expression of the calpain system is associated with poor clinical outcome in gastro-oesophageal adenocarcinomas. J Gastroenterol 48: 1213-1221, 2013.

15. Storr SJ, Thompson N, Pu X, Zhang Y and Martin SG: Calpain in breast cancer: Role in disease progression and treatment response. Pathobiology 82: 133-141, 2015.

16. Matena K, Boehm T and Dear N: Genomic organization of mouse Capn5 and Capn6 genes confirms that they are a distinct calpain subfamily. Genomics 48: 117-120, 1998.

17. Davis J, Martin SG, Patel PM, Green AR, Rakha EA, Ellis IO and Storr SJ: Low calpain-9 is associated with adverse disease-specific survival following endocrine therapy in breast cancer. BMC Cancer 14: 995, 2014.

18. Jorfi S, Ansa-Addo EA, Kholia S, Stratton D, Valley S, Lange $S$ and Inal J: Inhibition of microvesiculation sensitizes prostate cancer cells to chemotherapy and reduces docetaxel dose required to limit tumor growth in vivo. Sci Rep 5: 13006, 2015.

19. Kamarajan P, Hayami T, Matte B, Liu Y, Danciu T, Ramamoorthy A, Worden F, Kapila S and Kapila Y: Nisin ZP, a bacteriocin and food preservative, inhibits head and neck cancer tumorigenesis and prolongs survival. PLoS One 10: e0131008, 2015.

20. Rothe J, Wakileh M, Dreissiger K and Weber H: The flavonoid beverage Haelan 951 induces growth arrest and apoptosis in pancreatic carcinoma cell lines in vitro. BMC Complement Altern Med 15: 212, 2015.

21. Lee SJ, Choi YL, Lee EJ, Kim BG, Bae DS, Ahn GH and Lee JH: Increased expression of calpain 6 in uterine sarcomas and carcinosarcomas: An immunohistochemical analysis. Int J Gynecol Cancer 17: 248-253, 2007.

22. Dear TN and Boehm T: Diverse mRNA expression patterns of the mouse calpain genes Capn5, Capn6 and Capn11 during development. Mech Dev 89: 201-209, 1999.

23. Tonami K, Kurihara Y, Arima S, Nishiyama K, Uchijima Y, Asano T, Sorimachi H and Kurihara H: Calpain-6, a microtubule-stabilizing protein, regulates Racl activity and cell motility through interaction with GEF-H1. J Cell Sci 124: 1214-1223, 2011.

24. Hoskin V, Szeto A, Ghaffari A, Greer PA, Côté GP and Elliott BE: Ezrin regulates focal adhesion and invadopodia dynamics by altering calpain activity to promote breast cancer cell invasion. Mol Biol Cell 26: 3464-3479, 2015.

25. Lee SJ, Kim BG, Choi YL and Lee JW: Increased expression of calpain 6 during the progression of uterine cervical neoplasia: Immunohistochemical analysis. Oncol Rep 19: 859-863, 2008.

26. Rho SB, Byun HJ, Park SY and Chun T: Calpain 6 supports tumorigenesis by inhibiting apoptosis and facilitating angiogenesis. Cancer Lett 271: 306-313, 2008. 
27. Hong JM, Teitelbaum SL, Kim TH, Ross FP, Kim SY and Kim HJ: Calpain-6, a target molecule of glucocorticoids, regulates osteoclastic bone resorption via cytoskeletal organization and microtubule acetylation. J Bone Miner Res 26: 657-665, 2011.

28. Marion A, Dieudonnè FX, Patiño-Garcia A, Lecanda F, Marie PJ and Modrowski D: Calpain-6 is an endothelin-1 signaling dependent protective factor in chemoresistant osteosarcoma. Int J Cancer 130: 2514-2525, 2012.

29. Ivanova EV, Kondakova IV, Spirina LV, Afanas'ev SG, Avgustinovich AV and Cheremisina OV: Chymotrypsin-like activity of proteasomes and total calpain activity in gastric and colorectal cancer. Bull Exp Biol Med 157: 781-784, 2014.

30. Hanahan D and Weinberg RA: Hallmarks of cancer: The next generation. Cell 144: 646-674, 2011.
31. Hainaut $P$ and Plymoth A: Targeting the hallmarks of cancer: Towards a rational approach to next-generation cancer therapy. Curr Opin Oncol 25: 50-51, 2013.

32. Deeb D, Gao X, Liu YB, Pindolia K and Gautam SC: Pristimerin, a quinonemethide triterpenoid, induces apoptosis in pancreatic cancer cells through the inhibition of pro-survival Akt/NF- $\mathrm{B} / \mathrm{mTOR}$ signaling proteins and anti-apoptotic Bcl-2. Int J Oncol 44: 1707-1715, 2014.

33. Yoshida M, Miyasaka Y, Ohuchida K, Okumura T, Zheng B, Torata N, Fujita H, Nabae T, Manabe T, Shimamoto M, et al: Calpain inhibitor calpeptin suppresses pancreatic cancer by disrupting cancer-stromal interactions in a mouse xenograft model. Cancer Sci 107: 1443-1452, 2016.

34. Carragher NO: Calpain inhibition: A therapeutic strategy targeting multiple disease states. Curr Pharm Des 12: 615-638, 2006. 